\title{
Positional Nystagmus in Patients Evaluated for Dizziness and Imbalance
}

\author{
Richard A. Roberts, ${ }^{1}$ Samuel N. Bittel, ${ }^{2}$ and Richard E. Gans ${ }^{3}$ \\ ${ }^{1}$ Alabama Hearing \& Balance Associates, Inc., Foley, AL 36535, USA \\ ${ }^{2}$ Associated Audiologists, Shawnee Mission, KS 66204, USA \\ ${ }^{3}$ The American Institute of Balance, Largo, FL 33777, USA \\ Correspondence should be addressed to Richard A. Roberts; rroberts@hearingandbalance.net
}

Received 31 August 2015; Accepted 3 January 2016

Academic Editor: Sampath Prasad

Copyright (C) 2016 Richard A. Roberts et al. This is an open access article distributed under the Creative Commons Attribution License, which permits unrestricted use, distribution, and reproduction in any medium, provided the original work is properly cited.

\begin{abstract}
There is variability in the literature regarding the presence of positional nystagmus in healthy participants with reportedly normal vestibular and central nervous system function. This ranges from $7.5 \%$ to $88 \%$ and raises an important clinical question. If $88 \%$ of healthy participants have positional nystagmus then how is the clinician to interpret the presence of positional nystagmus in a patient presenting with dizziness and/or disequilibrium? The primary purpose of this investigation was to examine the prevalence and characteristics of positional nystagmus in patients evaluated specifically for dizziness and imbalance. Data was collected using retrospective chart review. 200 charts were randomly selected from all patients seen for evaluation of dizziness and imbalance over a period of eight months. Clinicians independently reviewed the data from positional testing for each chart. Nystagmus was present if there was a clear slow and fast phase component and there were three beats in a $10 \mathrm{~s}$ time window. Nystagmus direction and intensity data were collected. Results indicate positional nystagmus is present in $10.5 \%$ to $21 \%$ of patients evaluated for dizziness and imbalance. Use of liberal criteria for determining presence of positional nystagmus (i.e., 3 beats in 20 sec) may account for higher prevalence rates across other studies.
\end{abstract}

\section{Introduction}

Evaluation for positional nystagmus is an essential component of the vestibular and equilibrium evaluation $[1,2]$. This assessment subtest is sometimes referred to as static positional testing and is differentiated from Dix-Hallpike positioning maneuvers used to identify posterior and anterior canal benign paroxysmal positional vertigo (BPPV) $[1,2]$. During this test protocol, the clinician places the patient in different positions to look for provocation or modulation of nystagmus $[1,3]$. Common test positions include supine, head right and/or body right, and head left and/or body left positions. The primary reason for moving the patient into these positions is to alter the orientation of the labyrinth relative to the earth gravitational vector $[1,3]$. Changes in an existing spontaneous nystagmus or the provocation of nystagmus due to the position can occur because of central nervous system (CNS) involvement or peripheral vestibular involvement. Damage to the cerebellum, Arnold-Chiari malformation, multiple sclerosis, vertebrobasilar insufficiency, and even medication effects may all cause vertical positional nystagmus $[1,4,5]$. von Brevern et al. reported a variety of types of nystagmus observed in various static positions: geotropic, ageotropic, torsional, and downbeat for patients with definite migrainous vertigo [6]. We have reported on ageotropic horizontal nystagmus in patients with migrainous positional vertigo (MPV) [7].

The presence of positional nystagmus could also be an indicator of peripheral vestibular involvement. Of course, BPPV affecting the horizontal semicircular canals would be provoked using these types of positions $[8,9]$. Placing a patient with spontaneous nystagmus resulting from an acute unilateral labyrinthine involvement into various positions may also alter the nystagmus. Likewise, when restoration of vestibular tone occurs with static compensation, placing the patient into different positions with varying levels of 
neural input from the periphery may provoke a positional nystagmus since dynamic compensation may be incomplete [10]. This positional nystagmus of peripheral origin would be expected to be direction-fixed and horizontal with the fast phase of the nystagmus beating towards the intact ear consistent with Ewald's second law [3]. An irritative or recovery nystagmus which may deviate from this expectation and beat towards the involved ear is also possible [11].

While the presence of positional nystagmus has been addressed in normal controls $[12,13]$, there are few reports addressing the presence of positional nystagmus in patients with dizziness [14-16]. Bertholon et al. investigated the presence of positional nystagmus in 490 consecutive patients presenting to their facility with dizziness or imbalance [14]. In their study, both positioning (Dix-Hallpike) and static positions were considered. The Dix-Hallpike positioning test is used to assess patients for posterior semicircular canal BPPV. Interestingly, when the participants with posterior semicircular canal BPPV are excluded, these authors only observed positional nystagmus in $7.8 \%$ of their participants (38). These authors did not include mental tasking during their positional tests. On the other hand, these authors conducted their static positional testing with the patient's vision enabled and eyes fixated on the examiners face. Bertholon et al. state that they did this so that no "normal" nystagmus would be observed. How many of the 490 patients would have presented with positional nystagmus were this control not in place is unknown.

A second study which investigated positional nystagmus in patients with dizziness was completed by Aoki et al. [15]. The purpose of this investigation was to determine which of the three different static positions was the most effective in eliciting positional nystagmus. Participants completed a body only maneuver, a head only maneuver, and a head and body maneuver. Eye movement was recorded using electronystagmography (ENG) technology with eyes open under darkened goggles. From a total of 86 patients, 34 ( $40 \%)$ had positional nystagmus in at least one of these three positions. There is no discussion of mental alerting tasks.

Johkura et al. reported that $49 \%$ (98) of their participants with chronic dizziness had positional nystagmus while only $16.1 \%$ (25) of their controls had positional nystagmus [16]. As in the study by Bertholon et al., Johkura et al. also attempted to exclude participants with "normal" positional nystagmus. Prospective participants were excluded if positional nystagmus was observed using ordinary Frenzel goggles. There is no mention of the number of prospective participants excluded for this reason.

Although there are no other studies that specifically address the presence of positional nystagmus in a patient population with dizziness and imbalance, there are studies that have investigated nystagmus in other types of patient populations. For example, Kiyomizu et al. studied the presence of nystagmus in patients with diagnosed psychiatric problems using video-oculography [17]. Although their methodology is not clear in terms of the number of positions tested or use of mental tasking, the authors report that positional nystagmus was observed in only $21.1 \%$ (48) of cases out of 227 patients with psychiatric disease. Interestingly, the investigators also conducted similar testing in normal control participants and found positional nystagmus in only one of 107 participants, which amounts to $0.9 \%$.

Moubayed and Saliba report observing positional nystagmus in 50\% (13) of patients with vertebrobasilar insufficiency and also $50 \%$ (13) of patients with normal vertebral artery function [18]. Again, from the methodology it is not clear whether the researchers used mental tasking and also number and type of positions that were incorporated, but it is clear that the investigators used ENG testing.

From these studies, it appears that there is a range prevalence of positional nystagmus from a low of approximately $8 \%$ reported by Bertholon et al. to a high of $50 \%$ reported by Moubayed and Saliba. Of course, the specific methodology used to assess participants for positional nystagmus is not always clear from these reports. Another interesting consideration is the fact that there are several studies, which also report a wide range of positional nystagmus in the healthy normal population. These same studies range from a low of $1 \%$ when normal controls are considered to a high of $88 \%$ for studies specifically investigating positional nystagmus in healthy participants. It is certainly intriguing that some authors report up to $88 \%$ of patients of normal healthy patients, while investigators testing for positional nystagmus in patient populations report much lower rates.

The purpose of the current investigation is to report on the prevalence of positional nystagmus in patients seen for evaluation of dizziness and disequilibrium. Investigating this is important because there appears to be much variability in the literature. This is further complicated by the wide range of reported prevalence of positional nystagmus in the normal healthy population. This is problematic for the clinician when interpreting nystagmus in the patient population as normal or abnormal. We hypothesized that the prevalence of positional nystagmus in our patient population would be similar to that of Bertholon et al. (when posterior canal BPPV was excluded) and less than the higher values of $73-88 \%$ reported in the literature for normal, healthy participants.

\section{Methods}

2.1. Participants. A retrospective review of 200 charts from patients assessed at The American Institute of Balance was conducted. Approval was obtained by the Institutional Review Board of The American Institute of Balance prior to initiation of this investigation. The charts were selected from all of the charts from patients seen for vestibular and equilibrium evaluation. A table of random numbers was used to create a list used to select charts in as random a manner as possible. The data from 132 females and 68 males were included in this study with an age range from 21 to 93 years. Mean age was 67 years.

Although the focus of this investigation was on the results of static positional testing, it is important to acknowledge that all patients underwent comprehensive evaluation by audiologists with specific training in vestibular assessment and management. Standard assessment for all patients included detailed discussion of history and symptoms, rotary chair, VNG, vestibular evoked myogenic potential, and postural stability testing. Pertinent to this investigation, static 
positional testing was completed prior to bithermal caloric stimulation as some have observed that positional nystagmus is more likely following caloric testing [12]. For patients with a history consistent with possible uncompensated vestibular dysfunction, lateral headshake testing was also completed and dynamic visual acuity testing was completed, also. Auditory brainstem response was only performed on patients without recent imaging studies. Electrocochleography was only performed on patients with a history consistent with Meniere's disease. Standard audiometric results were also obtained. As common in most settings that conduct vestibular testing, all patients were asked to refrain from substances that are known to have the potential to influence evaluation results for 48 hours. This would include vestibular suppressant medications, alcohol, and nicotine.

2.2. Instrumentation. Binocular VNG recordings were obtained using a commercially available system to analyze, record, and quantify eye movements and nystagmus (balanceback; Kettering, OH, USA). This VNG system uses infrared cameras housed within goggles that contain nonlaser infrared emitting diodes. The infrared sources supply sufficient infrared "lighting" for high quality video imaging. During data collection for positional testing, a cover was kept in place that shielded the eyes of the patient from any light. This allowed testing to be completed in darkness but with eyes open. All eye movement video was recorded for the entire VNG exam and was available for replay if needed. Eye movement traces were printed and available in the chart of each patient. Positional testing was conducted using a typical examination table with a padded surface (Winco; Ocala, FL, USA). Dimensions of the examination table were $28^{\prime \prime} \times 72^{\prime \prime}$ and a height of $30^{\prime \prime}$.

2.3. Procedures. For all patients, static positional tests were performed during the VNG evaluation after oculomotor, gaze, and Hallpike but prior to caloric testing. Patients were positioned on the examination table with the VNG goggles in place and the cover on so that the patient was in total darkness (vision-denied). The patient was placed in the static positions as shown in Figures 1(a)-1(e): supine, head right, head left, body right, and body left. As shown in the figure, the head was positioned with the neck in slight flexion for supine. Also note the slight side flexion in body right and body left positioning. These positional tests were conducted in a vision-denied condition. Patients were held in each position for at least 15-20 seconds.

Once the charts were chosen using the random selection protocol, hard copies of the eye tracing data record for each positional test were analyzed. Analysis was completed individually by four audiologists with specific clinical expertise in vestibular and equilibrium assessment. All 200 records were reviewed by two examiners who were the first two authors of this investigation. Then, the subgroup of traces that the two authors identified as having positional nystagmus was reviewed by two additional audiologists. The data from ten patients with no positional nystagmus were also included in an attempt to observe any bias the additional audiologists might have to call normal eye movements nystagmus. Nystagmus was considered present if (1) there was an identifiable fast and slow component and (2) there were three beats of nystagmus observed in a $10 \mathrm{~s}$ time window. When the examiner observed nystagmus and the VNG system software also detected nystagmus, the slow phase velocity (SPV) in degrees per second $\left({ }^{\circ} / \mathrm{s}\right)$ calculated by the software algorithm was recorded. If the examiner identified nystagmus that was not detected by the software, the SPV was calculated by hand as with older strip-chart technology. Hand measurement was only necessary for four patients.

\section{Results}

The number of patients with positional nystagmus in at least one position is shown in Figure 2. For a given participant with positional nystagmus, the number of clinicians out of four possible who reported nystagmus as present is also shown. For 10 patients, only one or two of the clinicians identified positional nystagmus. For 32 patients, at least three or all four clinicians were in agreement that positional nystagmus was present. If this data is used to create a range from a conservative estimate of prevalence of positional nystagmus (all four clinicians had to observe the nystagmus) to a liberal estimate of prevalence of positional nystagmus (only one clinician had to observe the nystagmus), then the range is from 21 to 42 out of 200 patients. In other words, $10.5 \%$ to $21 \%$ of the patients had positional nystagmus. None of the normal traces were identified as having positional nystagmus.

Specific data regarding the 42 patients with positional nystagmus is shown in Table 1 . The reader should note that the interpretation provided in this table is based on history, symptoms, and overall assessment results, not just positional nystagmus data. Twenty-six of 132 female patients (19.7\%) and 16 of 68 male patients (23.5\%) had positional nystagmus. There was no difference in percentage of patients with positional nystagmus based on gender, $\chi^{2}(1, N=$ $200)=0.2, p=0.655$. Thirty-two of 42 patients $(76.2 \%)$ had nystagmus in only one or two positions. There was no correlation between patient age and number of positions with nystagmus $(r=0.037)$.

Only one patient had nystagmus in all five positions. The number of test positions with nystagmus is plotted in Figure 3. Although visual inspection suggests a trend for more frequent positional nystagmus in supine, head right, and head left position compared to body right and body left position, this did not reach statistical significance, $\chi^{2}(4$, $N=80)=4.63, p=0.328$. In Table 2 , it can be seen that direction-fixed nystagmus was observed significantly more often in patients compared to direction-changing nystagmus, $\chi^{2}(1, N=42)=9.52, p<0.05$. Horizontal nystagmus was more common than vertical nystagmus when the number of patients and the number of positions are considered. None of the patients had nystagmus that changed direction within a single position. Seven patients had nystagmus that had both vertical and horizontal component (mixed) or changed direction in different positions. Four patients exhibited an ageotropic nystagmus and none had a geotropic nystagmus. 


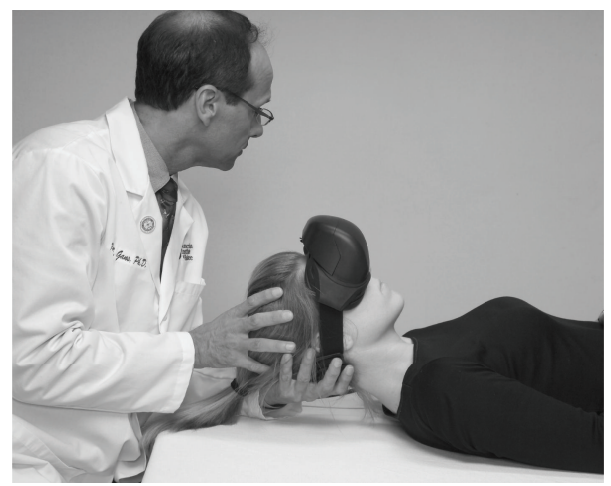

(a)

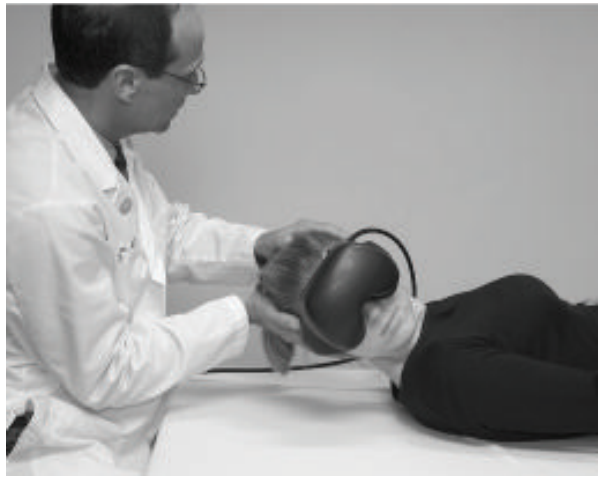

(b)

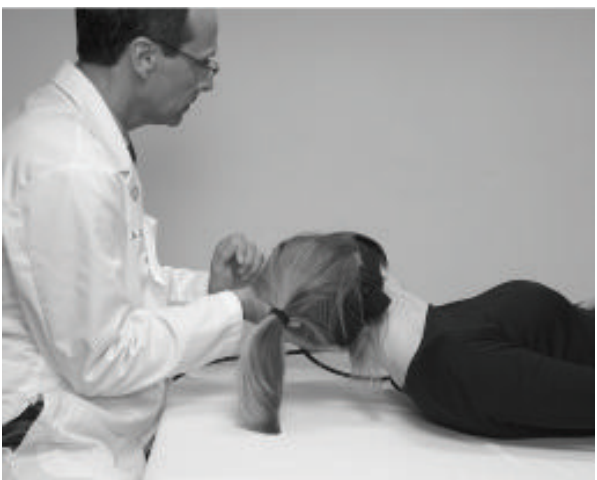

(d)

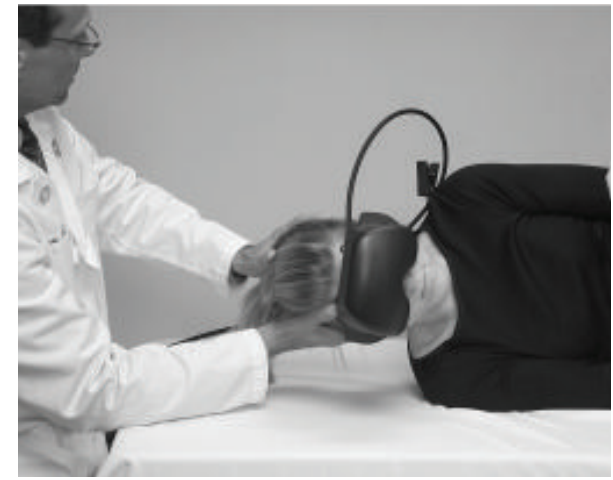

(c)

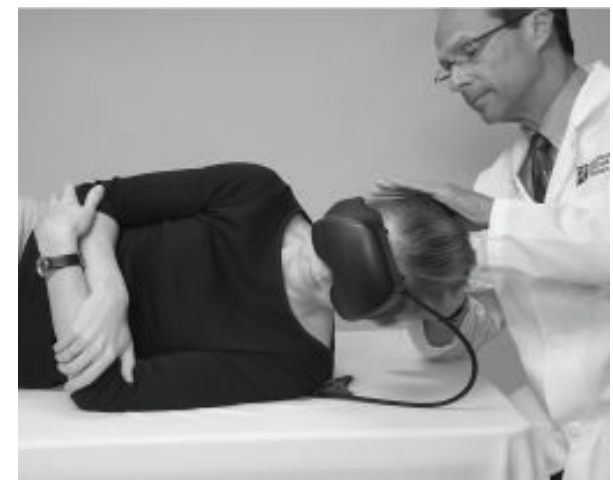

(e)

FIGURE 1: The five test positions are shown: (a) supine; (b) head right with $90^{\circ}$ rotation; (c) body right; (d) head left with $90^{\circ}$ rotation; (e) body left. Reprinted with permission from AIB Education Foundation Press.

The nystagmus mean slow phase velocity was $1.56^{\circ} / \mathrm{s}$ more intense for the direction-changing nystagmus compared to the direction-fixed nystagmus. This was not significantly different $(t=-1.84, \mathrm{df}=79$, and $p=0.06)$. When all nystagmus was considered, regardless of direction, the average slow phase velocity was $4.64^{\circ} /$ s.

When grouped by disorder, it is not clear that one position was more likely to elicit positional nystagmus. On the other hand, some patterns appear when nystagmus direction is considered as a function of disorder. This is shown in Figure 4. Patients with CNS involvement tended to have left-beating nystagmus. Those patients in the nonvestibular group more often had upbeating nystagmus. In the groups with unilateral BPPV, there was a pattern that left-beating nystagmus was observed more often with right posterior canal BPPV and right beating nystagmus was observed more often in patients with left posterior canal BPPV.

\section{Discussion}

4.1. Prevalence of Positional Nystagmus. Using a strict criterion that all four examiners had to independently identify nystagmus as present, the prevalence of positional nystagmus in our 200 patients with dizziness and imbalance was only $10.5 \%$. When the criterion was at its least restrictive, only one examiner had to identify positional nystagmus. Even using 


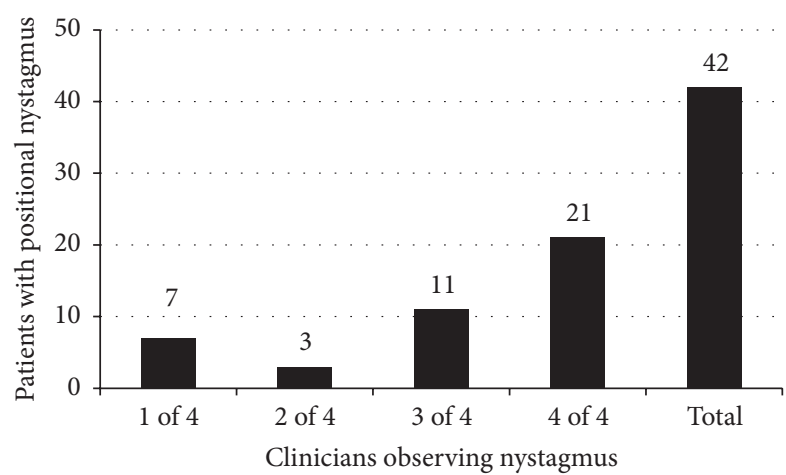

FIGURE 2: The number of patients with positional nystagmus in at least one position is grouped by the number of clinicians out of four who observed nystagmus. The total patients with positional nystagmus across these four groups are also provided.

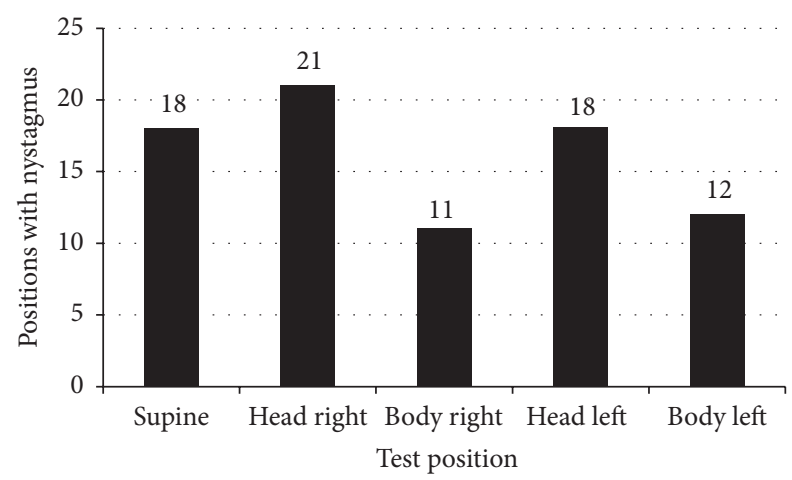

FIGURE 3: The number of test positions with observed nystagmus is shown grouped by test position.

this criterion, the prevalence was only $21 \%$ and this included vertical and horizontal nystagmus. This differs markedly from some reports $[12,13,19]$. Our results, however, are similar to prevalence rates reported by others for healthy participants $[16,20,21]$. Recall that Hajioff et al. only reported positional nystagmus in $7.5 \%$ of their healthy participants [20]. Hajioff et al. reported that $27 \%$ of their healthy participants had positional nystagmus [20] and Johkura et al. reported positional nystagmus in only $19 \%$ of their normal controls [16].

Using a participant group similar to the current study, Bertholon et al. reported that positional nystagmus was present in only $20 \%$ (100 of 490 ) of their patients referred for otoneurological examination [14]. This is close to our finding in a group of patients also referred for vestibular and equilibrium evaluation. A key difference is that we obtained data in a vision-denied condition where visual fixation was impossible. Bertholon et al. only included patients with observable positional nystagmus during visual fixation by the patient on the clinician's face. It is possible that more of their patients had positional nystagmus using their VNG technique with no visual fixation, but these were not discussed in the study.

Johkura et al. reported that $61 \%$ of their participants with chronic dizziness had positional nystagmus [16]. Although this is higher than the prevalence reported in our study and also by Bertholon et al., it is still much lower than that reported by others for participants without dizziness. Interestingly, Barber and Wright observed that positional nystagmus was more common if testing was performed after calorics compared to positional testing prior to caloric stimulation [12]. If the data of their participants who received caloric stimulation first is excluded, then the prevalence of positional nystagmus from their report decreases from $82 \%$ to $73 \%$. This result is identical to that of Sunami et al. [19] and at least moves closer to Johkura et al. [16] but is still higher than our results. This remains intriguing since we reiterate that Barber and Wright, as well as Sunami et al., used reportedly "normal" participants while Johkura et al. used patients with chronic dizziness.

One of the methodological differences between the current study and some others reporting a higher prevalence of positional nystagmus is that the patients were not mentally tasked during positional testing. A mental task such as asking patients to answer questions or perform simple arithmetic is often employed as a way to keep patients from suppressing nystagmus [22]. McAuley et al., as well as Barber and Wright, specifically state that mental alerting tasks were used to avoid suppression of nystagmus [12, 13]. It is not clear whether Sunami et al. used these procedures. At the opposite end of the prevalence data, Van der Stappen et al. and Hajioff et al. both used mental alerting tasks and had results similar to the current study [20,21]. Based on the literature, it is not clear that a lack of mental alerting explains our smaller prevalence of positional nystagmus.

The current investigation incorporated five specific test positions. Barber and Wright [12] and Sunami et al. used eight test positions [19]. One might argue that using a greater number of test positions may lead to a higher prevalence of positional nystagmus. However, McAuley et al. report the highest prevalence of positional nystagmus of all the studies reviewed and used only one more position (6) than the current study [13]. Hajioff et al. tested for positional nystagmus in nine different positions and only observed nystagmus in $27 \%$ of their participants [20]. The number of positions does not seem to be the main influencing factor on prevalence of positional nystagmus.

Given the variability among studies, one must also consider the methodology used to classify the presence of nystagmus. Several reports do not indicate this in their methodology [16, 19-21]. In the current study, the criteria for presence of nystagmus were as follows: (1) an identifiable fast and slow component and (2) three beats of nystagmus observed in a $10 \mathrm{~s}$ time window. Barber and Wright considered nystagmus as present when there were three consecutive beats with a recognizable slow and fast component [12]. They termed this a burst of nystagmus and then classified each burst as either "doubtful, possible nystagmus," "doubtful, probable nystagmus," or "unequivocal nystagmus." Within each of these categories, nystagmus was further classified as either persistent or intermittent. The authors report that positional nystagmus was observed in 397 of 888 positions tested. Yet, $40 \%$ of these instances of positional nystagmus were classified as either "doubtful, possible nystagmus" or 
TABLE 1: Age, gender, and overall interpretation are shown for each patient with nystagmus. Nystagmus direction is also provided with slow phase velocity $(\% / s)$ in parentheses.

\begin{tabular}{|c|c|c|c|c|c|c|c|}
\hline \multirow{2}{*}{ Age } & \multirow{2}{*}{ Gender } & \multicolumn{5}{|c|}{ Head/body positions } & \multirow{2}{*}{ Interpretation } \\
\hline & & Supine & Head right & Head left & Body right & Body left & \\
\hline 92 & $\mathrm{~F}$ & - & - & - & $\mathrm{L}(3)$ & - & CNS \\
\hline 78 & $\mathrm{M}$ & - & $\mathrm{L}(2)$ & - & - & - & PC-BPPV (R) \\
\hline 89 & $\mathrm{~F}$ & $\mathrm{D}(4)$ & - & - & - & - & $\mathrm{MD}$ \\
\hline 69 & $\mathrm{~F}$ & - & - & - & $\mathrm{L}(7)$ & $\begin{array}{l}\mathrm{R}(15) \\
\mathrm{D}(8)\end{array}$ & PC-BPPV (L) \\
\hline 83 & $\mathrm{~F}$ & - & $\mathrm{L}(6)$ & $\mathrm{R}(7)$ & - & - & PC-BPPV (R) \\
\hline 40 & M & - & $\mathrm{U}(4)$ & - & - & - & UVD (L) \\
\hline 39 & $\mathrm{~F}$ & - & - & - & $\mathrm{L}(1)$ & - & BVD \\
\hline 64 & M & - & - & - & - & $\mathrm{L}(4)$ & PC-BPPV (R), UVD (R) \\
\hline 54 & M & - & - & $\mathrm{L}(4)$ & - & - & HC-BPPV (L) \\
\hline 37 & $\mathrm{~F}$ & $\mathrm{U}(3)$ & $\mathrm{U}(3)$ & - & - & - & NV \\
\hline 67 & M & $\mathrm{L}(6)$ & - & - & - & - & $\mathrm{MD}$ \\
\hline 69 & $\mathrm{~F}$ & - & - & $\mathrm{R}(6)$ & $\mathrm{L}(1)$ & $\mathrm{R}(5)$ & PC-BPPV (L) \\
\hline 47 & $\mathrm{~F}$ & $\mathrm{U}(8)$ & - & $\mathrm{U}(2)$ & - & - & PC-BPPV (Bilateral) \\
\hline 82 & M & $\mathrm{R}(2)$ & - & - & - & - & PC BPPV (L) \\
\hline 57 & $\mathrm{~F}$ & $\mathrm{R}(4)$ & $\mathrm{R}(2)$ & - & - & - & UVD (L) \\
\hline 72 & $\mathrm{~F}$ & $\mathrm{~L}(5)$ & $\mathrm{L}(5)$ & $\mathrm{R}(5)$ & - & $\mathrm{R}(6)$ & CNS \\
\hline 66 & $\mathrm{~F}$ & $\mathrm{U}(4)$ & $\mathrm{U}(4)$ & - & - & - & Visual Preference \\
\hline 50 & $\mathrm{~F}$ & - & $\mathrm{U}(5)$ & $\mathrm{U}(4)$ & - & - & PC-BPPV (R) \\
\hline 81 & $\mathrm{~F}$ & - & - & - & $\mathrm{L}(5)$ & - & PC-BPPV (L) \\
\hline 76 & M & - & - & $\mathrm{R}(4)$ & - & - & PC-BPPV (L) \\
\hline 65 & M & - & $\mathrm{R}(2)$ & - & $\mathrm{R}(2)$ & - & PC-BPPV (L) \\
\hline 52 & $\mathrm{~F}$ & $\mathrm{U}(5)$ & $\mathrm{U}(6)$ & $\mathrm{U}(6)$ & - & - & NV \\
\hline 83 & $\mathrm{~F}$ & - & - & $\mathrm{L}(3)$ & - & - & PC-BPPV (R) \\
\hline 69 & $\mathrm{~F}$ & - & L (3) & $\mathrm{D}(5)$ & $\mathrm{L}(4)$ & $\mathrm{D}(3)$ & $\mathrm{MD}$ \\
\hline 45 & $\mathrm{~F}$ & - & - & - & $\mathrm{U}(20)$ & - & PC-BPPV (R) \\
\hline 63 & $\mathrm{~F}$ & - & - & $\mathrm{L}(2)$ & - & - & CNS \\
\hline 80 & $\mathrm{~F}$ & - & $\mathrm{L}(13)$ & $\mathrm{R}(3)$ & $\mathrm{L}(6)$ & $\mathrm{R}(4)$ & CNS \\
\hline 86 & M & - & - & - & - & $\mathrm{R}(6)$ & $\mathrm{MD}$ \\
\hline 87 & $\mathrm{~F}$ & $\mathrm{~L}(1)$ & $\mathrm{L}(5)$ & - & - & - & PC-BPPV (R) \\
\hline 71 & M & $\begin{array}{l}\mathrm{R}(1) \\
\mathrm{D}(5)\end{array}$ & $\begin{array}{l}\mathrm{R}(1) \\
\mathrm{D}(4)\end{array}$ & - & - & - & CNS \\
\hline 52 & $\mathrm{~F}$ & $\mathrm{D}(4)$ & L (5) D (4) & $\mathrm{R}(4)$ & - & - & Cervicogenic \\
\hline 75 & $\mathrm{~F}$ & L (3) & L (15) & $\mathrm{R}(12)$ & $\mathrm{L}(13)$ & $\mathrm{L}(8)$ & CNS \\
\hline 34 & $\mathrm{~F}$ & - & - & $\mathrm{L}(2)$ & - & - & High freq. vestibulopathy \\
\hline 64 & M & $\mathrm{R}(1)$ & $\mathrm{D}(2)$ & - & - & - & PC-BPPV (R) \\
\hline 70 & M & $\mathrm{U}(1)$ & $\mathrm{U}(1)$ & $\mathrm{U}(1)$ & - & - & $\mathrm{MD}$ \\
\hline 52 & M & $\mathrm{R}(3)$ & - & - & - & - & PC-BPPV (R) \\
\hline 75 & $\mathrm{~F}$ & $\mathrm{D}(1)$ & - & - & $\mathrm{D}(1)$ & $\mathrm{D}(1)$ & CNS \\
\hline 68 & M & - & - & - & - & $\mathrm{L}(1)$ & UVD (R) \\
\hline 70 & $\mathrm{~F}$ & - & $\mathrm{D}(18)$ & - & - & - & PC-BPPV (L) \\
\hline 78 & M & - & - & $\mathrm{D}(1)$ & - & - & $\mathrm{MD}$ \\
\hline 80 & M & - & - & - & - & $\mathrm{D}(6)$ & $\mathrm{NV}$ \\
\hline 66 & $\mathrm{~F}$ & - & $\mathrm{U}(2)$ & $\mathrm{R}(2)$ & - & - & NV \\
\hline
\end{tabular}

CNS, central nervous system involvement; D, down; F, female; HC-BPPV, horizontal canal benign paroxysmal positional vertigo; L, left; M, male; MD, multifactorial disequilibrium; NV, nonvestibular involvement; PC-BPPV, posterior canal benign paroxysmal positional vertigo; R, right; U, up; UVD, unilateral vestibular dysfunction. 
TABLE 2: Direction and intensity characteristics of positional nystagmus with $n=$ number of patients.

\begin{tabular}{|c|c|c|c|c|c|}
\hline \multirow{2}{*}{ Type } & \multirow{2}{*}{$(n)$} & \multirow{2}{*}{ Positions with nystagmus } & \multicolumn{3}{|c|}{ Nystagmus slow phase velocity $\left({ }^{\circ} / \mathrm{s}\right)$} \\
\hline & & & Range & Average & Standard deviation \\
\hline Direction-fixed & $(31)$ & 44 & $1-20$ & 3.93 & 3.79 \\
\hline Up & $(8)$ & 16 & $1-20$ & 4.81 & 4.51 \\
\hline Down & $(5)$ & 7 & $1-18$ & 4.57 & 6.24 \\
\hline Right & $(6)$ & 13 & $2-6$ & 3.13 & 1.46 \\
\hline Left & (12) & 8 & $1-6$ & 3.00 & 1.68 \\
\hline Direction-changing & (11) & 18 & $1-15$ & 5.49 & 3.77 \\
\hline Ageotropic & $(4)$ & 13 & $1-13$ & 5.54 & 2.73 \\
\hline Mixed & (3) & 11 & $1-15$ & 5.27 & 3.85 \\
\hline Up & & 1 & - & 2.00 & - \\
\hline Down & (4) & 3 & $2-5$ & 3.33 & 1.53 \\
\hline Right & $(x)$ & 3 & $2-12$ & 5.00 & 6.08 \\
\hline Left & & 6 & $3-15$ & 7.67 & 5.23 \\
\hline Overall & $(42)$ & 81 & $1-20$ & 4.64 & 3.84 \\
\hline
\end{tabular}

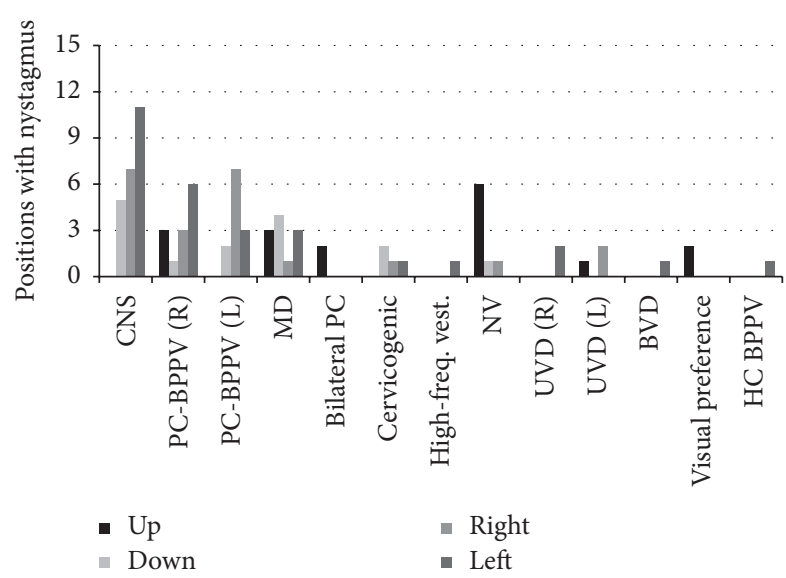

Figure 4: The number of test positions with nystagmus of each direction (up, down, right, and left) is shown grouped by patient diagnosis. CNS, central nervous system involvement; HC-BPPV, horizontal canal benign paroxysmal positional vertigo; L, left; MD, multifactorial disequilibrium; NV, nonvestibular involvement; PC$\mathrm{BPPV}$, posterior canal benign paroxysmal positional vertigo; $\mathrm{R}$, right; UVD, unilateral vestibular dysfunction.

"doubtful, probable nystagmus." Within these two categories, the nystagmus was considered intermittent $75 \%$ of the time when classified as "doubtful, possible nystagmus" and $77 \%$ of the time when classified as "doubtful, probable nystagmus."

In McAuley et al. work, the authors considered nystagmus as present when a minimum of three beats occurred within $20 \mathrm{sec}$ [13]. The nystagmus was then classified as either persistent or intermittent. Persistent nystagmus had to be present in $80 \%$ of the recording window. All other nystagmus cases were considered intermittent as long as there were three beats within $20 \mathrm{sec}$. Using these criteria, 51\% of their participants were identified as having persistent nystagmus in at least one position while $78 \%$ were identified as having intermittent nystagmus in at least one position.
It is possible that we avoided classifying ambiguous eye movements as nystagmus in the current study by adopting more strict criteria for nystagmus presence and by using a binary system of nystagmus either present or absent. This may have led to a lower overall prevalence of positional nystagmus in our patient group.

4.2. Effects of Gender and Age. Regarding gender, we observed a similar prevalence of $19.7 \%$ for female participants and $23.5 \%$ for male patients. McAuley et al. of course reported higher overall prevalence of positional nystagmus but similar rates between the genders [13]. They report that $92 \%$ of females and $84 \%$ of males had positional nystagmus. Other studies included both genders, but their reports do not allow for evaluation of differences on this parameter. Based on our results and those of McAuley et al. we do not expect the presence of positional nystagmus to vary with gender.

We did not observe a relationship between age and incidence of positional nystagmus in the current study. This was not specifically tested in other studies of positional nystagmus. Sunami et al. report that their participants ranged in age from 25 to 40 years (mean age $=25$ years) and had a positional nystagmus prevalence of $73 \%$ [19]. No age range is provided in Van der Stappen et al. but the mean age of their participants was 45 years and they observed positional nystagmus in $7.5 \%$ [21]. The group in Hajioff et al. was more similar in age to the current study with all participants over the age of 65 [20]. Their prevalence was 27\% while Johkura et al. observed positional nystagmus in $19 \%$ of their participants who ranged in age from 40 to 90 years (mean 71.8 years) [16]. McAuley et al. provide a range of ages from 20 to 70 but there are no measures of central tendency [13]. There does not appear to be a consistent, if any, relationship between prevalence of positional nystagmus and age.

4.3. Effects of Position. Specific data on test positions is not available in all studies. Most of the patients in the current study with positional nystagmus only had this in one or two test positions $(76.2 \%$; 32 of 42$)$. One patient had nystagmus 
in all five test positions. This differs from Sunami et al. whose participants tended to have nystagmus in more positions [19]. Only $29 \%$ of their participants had nystagmus in one or two test positions. They report that $11 \%$ (7 of 65 ) actually had nystagmus in all eight of their test positions. From Table 1 of the paper by McAuley et al., 30\% (13 of 43) of their participants with positional nystagmus had either intermittent or persistent nystagmus in all six test positions [13]. Only $14 \%$ of these participants had nystagmus in one or two positions. Based on these data, there is disparity between studies not only in terms of overall prevalence of positional nystagmus but also in the frequency across positions when it is observed.

There was no significant effect of test position in our study. McAuley et al. also indicate that their distribution of nystagmus was similar for all positions [13]. It is interesting that in our study $50 \%$ (21 of 42 ) of patients had nystagmus in head right position. $43 \%$ (18 of 42 ) had nystagmus in supine position and $43 \%$ also had nystagmus in head left position. This agrees with Sunami et al. for head right position who found nystagmus in 51\% (33 of 65) of participants [19]. Their prevalence rates were slightly lower for supine and higher for head left position. Also, Sunami et al. report the highest rates of nystagmus with lateral positioning to the right $(63 \%$; 41 of $65)$ and left (58\%; 38 of 65) [19].

4.4. Nystagmus Direction and Intensity. In the current study, the direction-fixed positional nystagmus was observed almost three times more often $(73.8 \%)$ than directionchanging nystagmus $(26.2 \%)$. This is very similar to the proportions reported by McAuley et al. for positional nystagmus in the horizontal plane [13]. McAuley et al. reported that $70 \%$ of their participants had a direction-fixed nystagmus and $30 \%$ had a direction-changing nystagmus. Sunami et al. still reported that most participants had direction-fixed nystagmus but this accounted for $46.2 \%$ of all the participants with positional nystagmus [19].

When positional nystagmus was present, it was more likely to occur in the horizontal plane than in the vertical plane. Only four participants in Sunami et al. had vertical nystagmus [19]. It is noted that these authors reported torsional and mixed nystagmus in $31 \%$ (20 of 65 ) of participants. Although we were unable to observe purely torsional nystagmus in the current study, patients with recordable vertical and horizontal components were included. It is likely that some of these patients would have been included in the mixed group of Sunami et al., bringing our data into closer agreement. This may also suggest that the relative prevalence of horizontal versus vertical positional nystagmus is probably not as different as suggested in their data when only these two categories are considered.

Overall, there is agreement among most studies of positional nystagmus regarding intensity. The average slow phase velocity in the current investigation was $4.64^{\circ} / \mathrm{s}$. We found no difference in intensity for participants with direction-fixed compared to those with direction-changing nystagmus. Van der Stappen et al. reported that one of their participants had a $6^{\circ} / \mathrm{s}$ positional nystagmus and two had slow phase velocities (SPVs) of $4^{\circ} / \mathrm{s}$ [21]. Averaged together, this is $4.67^{\circ} / \mathrm{s}$. Johkura et al. report mean SPVs of $0.79-4.01^{\circ} / \mathrm{s}$ [16]. McAuley et al. report a mean SPV of $5.04^{\circ} / \mathrm{s}[13]$.

4.5. Nystagmus by Diagnosis. The main purpose of this investigation was not to determine the types of positional nystagmus observed with different diagnoses. Our patients were not divided evenly by diagnosis and we recognize the limitation of describing nystagmus characteristics for specific groups when only small samples are available. However, some interesting patterns do emerge when we consider nystagmus direction. For our patients with posterior canal BPPV and nystagmus in static positions, there was a tendency for the nystagmus to beat away from the involved ear. Rotary nystagmus during positioning testing (Dix-Hallpike) is characteristic but the presence of positional nystagmus is not appreciated in the literature. One explanation for the presence of this type of positional nystagmus may be that the brainstem is attempting to compensate for the abnormal neural activity from the involved side during provocation of symptoms. This could lead to inhibition of neural activity from the involved side leading to a brainstem-level asymmetry favoring the unaffected side during certain positions. This would be expected based on Ewald's law with the fast phase of a nystagmus response beating towards the side with greater neural activity (healthy ear). Jacobson et al. provide an excellent description of the process of recovery nystagmus but central compensation is explained nicely also [11].

Adding further support, of the 16 patients with unilateral BPPV, 13 were specifically checked for positional nystagmus after treatment of BPPV. Eleven of these had no positional nystagmus after the BPPV was cleared by repositioning maneuvers. This may support that the nystagmus observed in static positional testing for these individuals was related to a compensation process at the brainstem level in response to the abnormal neural activity ultimately due to the presence of otoconial debris in the affected semicircular canal. Once the debris is cleared, the brainstem neural activity returns to normal and the positional nystagmus is no longer present.

Four of our patients had findings that were inconsistent with vestibular involvement. Three of these patients had upbeating nystagmus in at least one position and the fourth had a downbeating nystagmus. Vertical nystagmus is often reported with CNS involvement or pharmacologic influence $[3,23]$. In these four cases, a specific diagnosis could not be determined except that the results did not appear to be consistent with vestibular dysfunction.

Our patients with positional nystagmus who were diagnosed with CNS involvement most often had a left-beating nystagmus. Vertical nystagmus is common with CNS involvement [23], but horizontal nystagmus is also reported [6, 7]. Five of these seven patients had either a direction-changing positional nystagmus or vertical nystagmus. These findings are often reported with CNS involvement or pharmacologic (positional alcohol nystagmus) effects. Only two of these patients had horizontal nystagmus in a single position and in both cases this was a left-beating nystagmus which could be related to the location of their lesion. It is interesting to note that McAuley et al. reported that of their participants with direction-fixed nystagmus $47 \%$ had a leftward fast phase 
compared to only $23 \%$ with a rightward fast phase [13]. No explanation is given for this. It is interesting that Coats considered this enough to have potentially attributed such a finding to an imprinted motor pattern possibly created by reading [24].

\section{Conclusions}

Historically, many clinicians have held that a certain prevalence of "normal" positional nystagmus exists in the healthy population. This is supported in the literature by several studies in patients with reportedly intact vestibular and CNS function $[12,13,19,24]$. These studies indicate that $73-88 \%$ of patients with no reported health issues have positional nystagmus. There is also a growing body of literature indicating that positional nystagmus is not as common in healthy patients $[16,20,21]$, ranging from only $7.5 \%$ to $27 \%$. The results of our investigation are in agreement with the latter cited investigations and suggest that positional nystagmus may only be present in $10.5 \%$ to $21 \%$ of patients evaluated for dizziness and imbalance.

\section{Abbreviations \\ BPPV: Benign paroxysmal positional vertigo \\ CNS: Central nervous system \\ ENG: Electronystagmography \\ MPV: Migrainous positional vertigo \\ s: $\quad$ Seconds \\ SPV: Slow phase velocity \\ VNG: Videonystagmography.}

\section{Conflict of Interests}

The authors declare that there is no conflict of interests regarding the publication of this paper.

\section{Acknowledgments}

The authors are grateful to Patricia Harrington, Au. D., and Kenton S. Tarver, Au. D., for examining patient data traces for the presence of positional nystagmus.

\section{References}

[1] T. Brandt, "Positional and positioning vertigo and nystagmus," Journal of the Neurological Sciences, vol. 95, no. 1, pp. 3-28, 1990.

[2] R. Gans and W. Yellin, "Assessment of vestibular function," in Audiology: Diagnosis, R. Roeser, M. Valente, and H. HosfordDunn, Eds., pp. 540-566, Thieme, New York, NY, USA, 2007.

[3] R. Roberts and R. Gans, "Background, technique, interpretation, and usefulness of positional/positioning testing," in Balance Function Assessment and Management, G. Jacobson and N. Shepard, Eds., pp. 171-196, Plural Publishing Inc, San Diego, Calif, USA, 2008.

[4] R. Baloh and V. Honrubia, Clinical Neurophysiology of the Vestibular System, Oxford University Press, New York, NY, USA, 3rd edition, 2001.
[5] R. Leigh and D. Zee, The Neurology of Eye Movements, Oxford University Press, New York, NY, USA, 3rd edition, 2001.

[6] M. von Brevern, A. Radtke, A. H. Clarke, and T. Lempert, "Migrainous vertigo presenting as episodic positional vertigo," Neurology, vol. 62, no. 3, pp. 469-472, 2004.

[7] R. A. Roberts, R. E. Gans, and A. H. Kastner, "Differentiation of migrainous positional vertigo (MPV) from horizontal canal benign paroxysmal positional vertigo (HC-BPPV)," International Journal of Audiology, vol. 45, no. 4, pp. 224-226, 2006.

[8] R. Baloh, "Horizontal benign positional vertigo," Neurology, vol. 44, article 2214, 1994.

[9] G. C. Appiani, G. Catania, and M. Gagliardi, "A liberatory maneuver for the treatment of horizontal canal paroxysmal positional vertigo," Otology and Neurotology, vol. 22, no. 1, pp. 66-69, 2001.

[10] D. Zee, "Vestibular adaptation," in Vestibular Rehabilitation, S. Herdman, Ed., pp. 77-87, F. A. Davis Company, Philadelphia, $\mathrm{Pa}$, USA, 2nd edition, 2000.

[11] G. P. Jacobson, R. Pearlstein, J. Henderson, J. H. Calder, and J. Rock, "Recovery nystagmus revisited," Journal of the American Academy of Audiology, vol. 9, no. 4, pp. 263-271, 1998.

[12] H. Barber and G. Wright, "Positional nystagmus in normals," Advances in Oto-Rhino-Laryngology, vol. 19, pp. 276-285, 1973.

[13] J. R. McAuley, J. D. Dickman, W. Mustain, and V. K. Anand, "Positional nystagmus in asymptomatic human subjects," Otolaryngology-Head and Neck Surgery, vol. 114, no. 4, pp. 545-553, 1996.

[14] P. Bertholon, S. Tringali, M. B. Faye, J. C. Antoine, and C. Martin, "Prospective study of positional nystagmus in 100 consecutive patients," Annals of Otology, Rhinology and Laryngology, vol. 115, no. 8, pp. 587-594, 2006.

[15] S. Aoki, Y. Arai, and N. Kikuchi, "An effective maneuver of positional test by turning of the head and body together," Auris Nasus Larynx, vol. 35, no. 1, pp. 37-40, 2008.

[16] K. Johkura, T. Momoo, and Y. Kuroiwa, "Positional nystagmus in patients with chronic dizziness," Journal of Neurology, Neurosurgery and Psychiatry, vol. 79, no. 12, pp. 1324-1326, 2008.

[17] K. Kiyomizu, K. Matsuda, K. Torihara et al., "Nystagmus using video-oculography in psychiatric patients," European Archives of Oto-Rhino-Laryngology, vol. 266, no. 8, pp. 1167-1174, 2009.

[18] S. P. Moubayed and I. Saliba, "Vertebrobasilar insufficiency presenting as isolated positional vertigo or dizziness: a doubleblind retrospective cohort study," Laryngoscope, vol. 119, no. 10, pp. 2071-2076, 2009.

[19] K. Sunami, R. Tochino, T. Zushi et al., "Positional and positioning nystagmus in healthy subjects under videonystagmoscopy," Acta Oto-Laryngologica. Supplementum, vol. 554, pp. 35-37, 2004.

[20] D. Hajioff, R. M. Barr-Hamilton, N. R. Colledge, S. J. Lewis, and J. A. Wilson, "Re-evaluation of normative electronystagmography data in healthy ageing," Clinical Otolaryngology \& Allied Sciences, vol. 25, no. 4, pp. 249-252, 2000.

[21] A. Van der Stappen, F. L. Wuyts, and P. H. Van de Heyning, "Computerized electronystagmography: normative data revisited," Acta Oto-Laryngologica, vol. 120, no. 6, pp. 724-730, 2000.

[22] H. O. Barber and G. Wright, "Release of nystagmus suppression in clinical electronystagmography," Laryngoscope, vol. 77, no. 6, pp. 1016-1027, 1967. 
[23] T. Brandt, Vertigo: Its Multisensory Syndromes, Springer, London, UK, 1991.

[24] A. C. Coats, "Computer-quantified positional nystagmus in normals," American Journal of Otolaryngology-Head and Neck Medicine and Surgery, vol. 14, no. 5, pp. 314-326, 1993. 


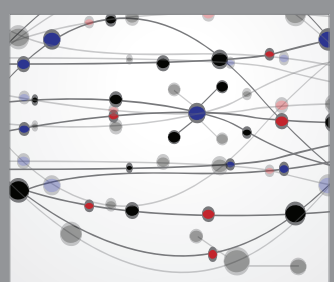

The Scientific World Journal
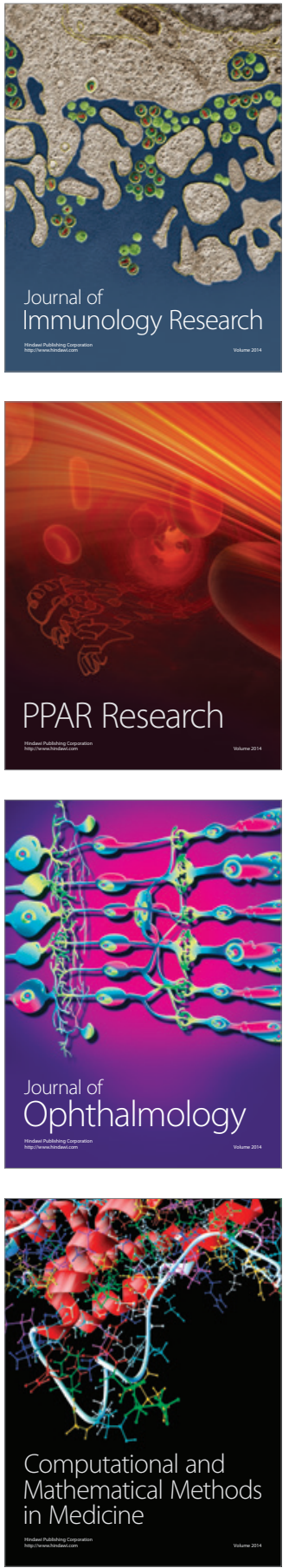

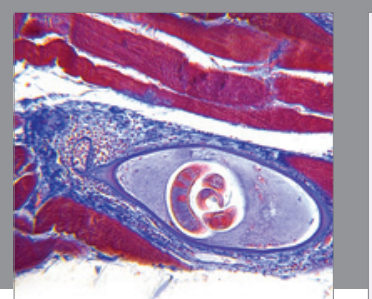

Gastroenterology Research and Practice

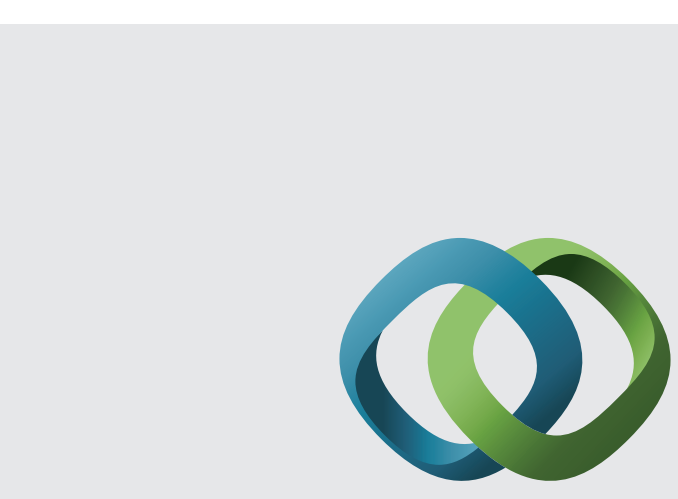

\section{Hindawi}

Submit your manuscripts at

http://www.hindawi.com
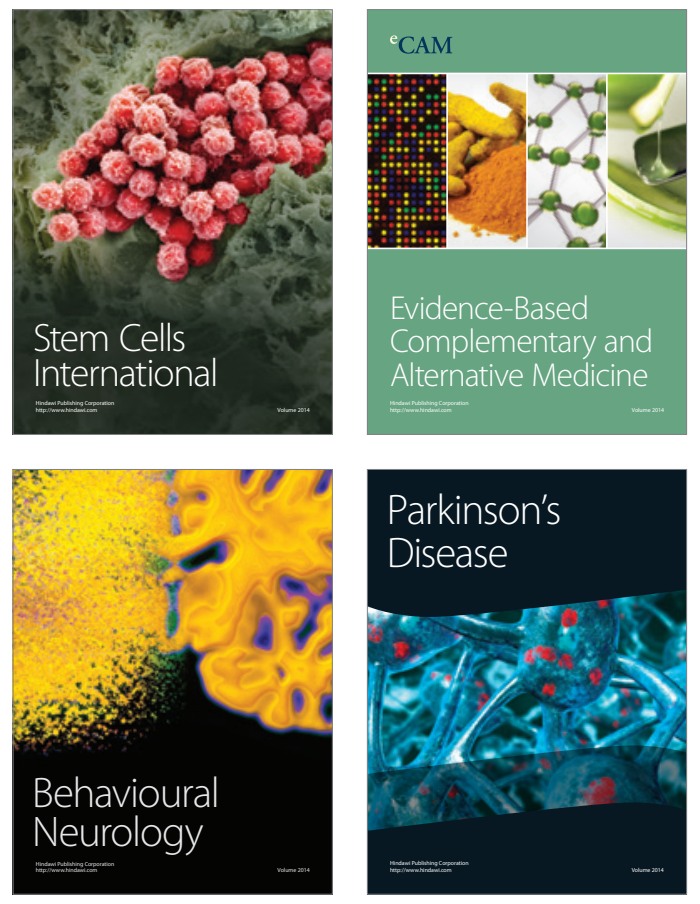
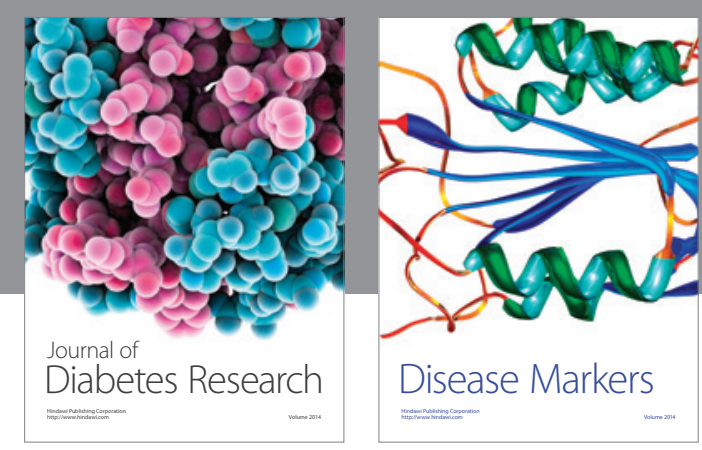

Disease Markers
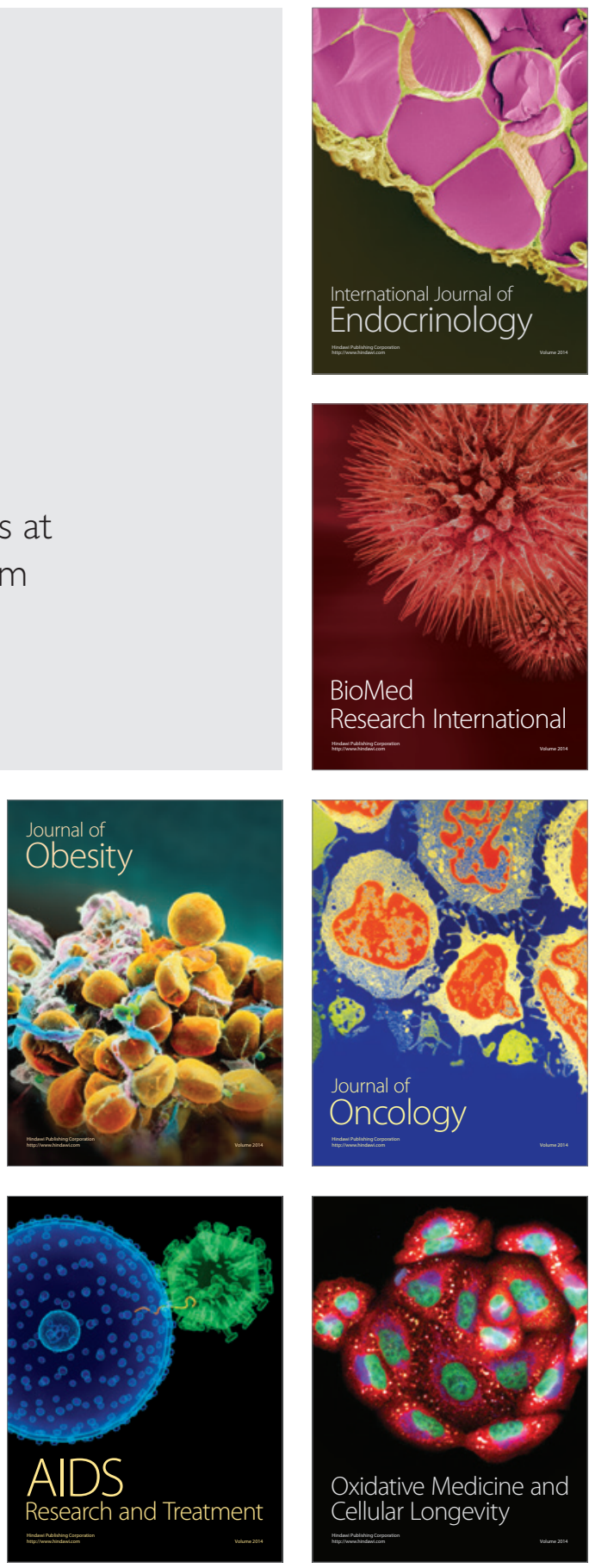